\title{
Non-rigid Registration with Missing Correspondences in Preoperative and Postresection Brain Images ${ }^{\star}$
}

\author{
Nicha Chitphakdithai ${ }^{1}$ and James S. Duncan ${ }^{1,2,3}$ \\ Departments of \\ ${ }^{1}$ Biomedical Engineering \\ 2 Electrical Engineering \\ ${ }^{3}$ Diagnostic Radiology \\ Yale University, New Haven, CT, USA \\ nicha.chitphakdithai@yale.edu
}

\begin{abstract}
Registration of preoperative and postresection images is often needed to evaluate the effectiveness of treatment. While several nonrigid registration methods exist, most would be unable to accurately align these types of datasets due to the absence of tissue in one image. Here we present a joint registration and segmentation algorithm which handles the missing correspondence problem. An intensity-based prior is used to aid in the segmentation of the resection region from voxels with valid correspondences in the two images. The problem is posed in a maximum a posteriori (MAP) framework and optimized using the expectation-maximization (EM) algorithm. Results on both synthetic and real data show our method improved image alignment compared to a traditional non-rigid registration algorithm as well as a method using a robust error kernel in the registration similarity metric.
\end{abstract}

\section{Introduction}

In localization-related epilepsy, brain resection is often considered the next line of treatment if anticonvulsive medication is unable to control seizures [1. To better understand epilepsy and the changes that occur in the brain after resection, preoperative and postresection images first need to be aligned. This registration problem is challenging due to the missing correspondences caused by resected tissue coupled with the possibility for highly nonlinear deformations in the postresection brain.

While many traditional non-rigid registration algorithms are available, few handle the problem of missing correspondences. A method for general registration problems was presented in 2] which dealt with partial data by using an EM-style method to simultaneously estimate the registration parameters and the missing data. Each voxel is assumed to have an equal chance of being labeled as missing or valid data. For our application, it is known that resection

\footnotetext{
^ This work was supported by NIH 5R01EB000473-08.
} 
voxels will appear dark in T1-weighted MRI due to cerebrospinal fluid (CSF) taking place of the missing tissue [3], and we will thus incorporate this prior information.

Some application-specific methods have also been proposed to better register images with substantial changes. The correspondence problem in the alignment of dynamic contrast-enhanced breast MRI was handled by "de-enhancing" the contrast images before registration in 44. Biomechanical models of brain tumor growth and estimation of tissue loss and replacement have been used to improve the registration accuracy around the tumor region 5. An algorithm to accommodate resection and retraction was presented in [6] for alignment of preoperative to intraoperative brain images. Their method alternated between registering the images using a demons algorithm with an anisotropic diffusion smoother and segmenting the resection by evolving a level set in the region with high intensity errors. We are proposing a probabilistically-grounded approach to the simultaneous registration and resection estimation problem.

In this paper, we present a joint registration and segmentation method in which an indicator function separating the valid and missing correspondence regions is estimated along with the transformation parameters. The addition of the indicator map into the registration estimation allows the incorporation of different models for observing the data under different correspondence assumptions. The general joint registration and segmentation framework follows the method described in [7. Previously in 8] we used a spatial prior on the valid tissue and resection locations to help estimate these regions. Due to the difficulty in gathering a training set for the spatial prior, we now explore a prior on postresection image intensities. Posing the problem in a MAP framework allows for the natural inclusion of an intensity-based prior.

\section{Methods}

\subsection{Registration and Segmentation Framework}

The goal of the registration problem is to determine the optimal transformation $T$ which aligns voxel $\mathbf{x}$ in the preoperative image $P$ with voxel $T(\mathbf{x})$ in the postresection image $R$. We incorporate a "hidden" indicator map $I$ which gives the segmentation of valid tissue in the postresection image $R$ from the resection region. The registration problem is then posed in a marginalized MAP framework, where we solve for

$$
\hat{T}=\underset{T}{\arg \max } \log \sum_{I} p(T, I \mid P, R)
$$

by using the EM algorithm. In the $(k+1)$ th iteration, the E-step gives an estimate for the indicator map given the estimate of the transformation $T^{k}$ from the previous iteration. The M-step then determines the new registration parameters $T^{k+1}$ using the information from the E-step. The algorithm keeps iterating between the E-step and M-step until the registration parameters converge. 
In our instance of the EM algorithm, the M-step can be written as

$$
\begin{gathered}
T^{k+1}=\underset{T}{\arg \max } E_{I \mid P, R, T^{k}}[ \\
\log p(P \mid R, I, T)+\log p(R \mid I, T) \\
+\log p(I \mid T)+\log p(T)] .
\end{gathered}
$$

We next make a few simplifications. First we make the common assumption of independence of voxels in the image. We also assume that given the indicator, the postresection image does not depend on $T$. To simplify (2) further, we assume no prior spatial information for the indicator map. We can then rewrite (2) as

$$
\begin{gathered}
T^{k+1}=\underset{T}{\arg \max } \sum_{\mathbf{x} \in R} \sum_{l \in L} p\left(I(\mathbf{x})=l \mid P, R, T^{k}\right)[\log p(P(T(\mathbf{x})) \mid R, I(\mathbf{x})=l, T) \\
+\log p(R(\mathbf{x}) \mid I(\mathbf{x})=l)]+\log p(T)
\end{gathered}
$$

where $L$ is the set of possible labels in the indicator map. In the following we use $L=\{0,1,2\}: I(\mathbf{x})=0$ for the resection, $I(\mathbf{x})=1$ for a voxel with a valid correspondence, and $I(\mathbf{x})=2$ for a background voxel.

The E-step calculates the probability that a voxel is assigned a certain label. Using Bayes' rule, this probability can be computed from

$$
p\left(I(\mathbf{x})=l \mid P, R, T^{k}\right)=\frac{p\left(P\left(T^{k}(\mathbf{x})\right) \mid R, I(\mathbf{x})=l, T^{k}\right) p(R(\mathbf{x}) \mid I(\mathbf{x})=l)}{\sum_{l^{\prime}} p\left(P\left(T^{k}(\mathbf{x})\right) \mid R, I(\mathbf{x})=l^{\prime}, T^{k}\right) p\left(R(\mathbf{x}) \mid I(\mathbf{x})=l^{\prime}\right)}
$$

After the EM algorithm converges, the final indicator map $\hat{I}$ is estimated from these probabilities by assigning $\hat{I}(\mathbf{x})=\underset{l}{\arg \max } p(I(\mathbf{x})=l \mid P, R, \hat{T})$.

\subsection{Probability Models}

To calculate (3) and (4), we need to define probability models for the similarity measure $p(P(T(\mathbf{x})) \mid R, I(\mathbf{x})=l, T)$, the prior on intensities given the indicator function value $p(R(\mathbf{x}) \mid I(\mathbf{x})=l)$, and the transformation prior $p(T)$.

Similarity Term. The probability $p(P(T(\mathbf{x})) \mid R, I(\mathbf{x})=l, T)$ acts like the similarity metric of a standard registration algorithm. For different values of the indicator map, we can use a different probability model to describe how we expect the images to match. We assume a voxel labeled as part of the resection in $R$ can match any intensity in $P$ with equal probability and use a uniform distribution. When a voxel is labeled as having a valid correspondence, the intensities should match, so we model $P(T(\mathbf{x})) \mid R, I(\mathbf{x})=l, T \sim N\left(R(x), \sigma_{1}\right)$. Finally, for a background voxel, we again employ a normal distribution but assign a larger standard deviation since we are not actually interested in matching the background. To summarize, the models under each indicator label are 


$$
p(P(T(\mathbf{x})) \mid R, I(\mathbf{x})=l, T)=\left\{\begin{array}{cl}
1 / c \quad, & l=0 \\
\frac{1}{\sqrt{2 \pi} \sigma_{1}} \exp \left(-\frac{[P(T(\mathbf{x}))-R(\mathbf{x})]^{2}}{2 \sigma_{1}^{2}}\right), & l=1 \\
\frac{1}{\sqrt{2 \pi} \sigma_{2}} \exp \left(-\frac{[P(T(\mathbf{x}))-R(\mathbf{x})]^{2}}{2 \sigma_{2}^{2}}\right), & l=2
\end{array}\right.
$$

where $c$ is the number of intensity levels in each image and $\sigma_{1}<\sigma_{2}$. For the experiments in this paper, we set $\sigma_{2}=2 \sigma_{1}$.

Intensity Prior. The term $p(R(\mathbf{x}) \mid I(\mathbf{x})=l)$ is the intensity-based prior in which given the indicator map label, we assume some knowledge of the intensities in the postresection image. For a voxel labeled as resection, we model its intensity using a normal distribution with a maximum likelihood estimate of the mean and standard deviation from manually segmented resection regions from a training set of postoperative images. If a voxel is labeled as having a valid correspondence, we assume any intensity in $R$ is equally likely to have a matching correspondence in $P$ and use a uniform distribution. Finally, background voxels are assumed to follow a normal distribution with 0 mean and a small standard deviation.

Transformation Prior. We use free form deformations (FFD) based on uniform cubic B-splines to model the non-rigid transformation as in [9]. The transformation parameters are then the B-spline control points $\mathbf{t}_{i}$, spaced $\delta$ apart. We assume the control points and its components $t_{i, j}$ are independent so that $p(T)=\prod_{i} \prod_{j} p\left(t_{i, j}\right)$, where $p\left(t_{i, j}\right)$ follows a normal distribution with mean equal to the starting location of the control point component on the uniform grid and standard deviation equal to $\frac{0.4 \delta}{3}$ to encourage a smooth transformation. In addition, we enforce a hard constraint to ensure the transformation is injective by restricting the control points to lie within a sphere of radius $0.4 \delta$ [10].

\subsection{Registration Methods for Comparison}

We compare our joint registration and indicator map estimation (RIME) method to a standard non-rigid registration (SNRR) method found in BioImage Suite (BIS) software [11. In addition, we compare an algorithm using a robust squared error in the similarity measure, i.e. $\rho(R(\mathbf{x})-P(T(\mathbf{x}))) \sim N(0, \sigma)$, where

$$
\rho(x)=\left\{\begin{array}{cl}
\frac{s^{2}}{6}\left(1-\left(1-\frac{x}{s}\right)^{2}\right)^{3} & ,|x| \leq s \\
\frac{s^{2}}{6} & ,|x|>s
\end{array},\right.
$$

is the Tukey function with scaling parameter $s[12$. The influence of outliers is reduced by mapping errors greater than $s$ to a maximum value. In this way, the intensity disparity in the resection region is given less influence in determining the optimal transformation. We automatically estimate $s=3 \sigma$, where $\sigma$ is the standard deviation of the intensity differences for the current overlap of images.

Both SNRR and robust Tukey registration (RTR) use a FFD transformation model with the same control point spacing as in RIME. In addition, note that all methods utilize a similarity measure based on direct intensity differences. 


\section{Results}

\subsection{Synthetic Images}

We used 11 pairs of $2 \mathrm{D}$ synthetic MR images. For each pair, a slice from a normal real 3D MRI was taken as the preoperative image. The postoperative image was created by first assigning resection voxels normally distributed random intensities with mean and standard deviation from a CSF sample from the preoperative image. The image was then warped by a physical model. The deformation simulations, while not necessarily mimicking true non-rigid motion after resection, provide a ground truth of displacement vectors at each voxel. The intensity prior for the image to be registered was trained on the remaining postresection images.

Figure 1 shows example results. Difference images were masked by the true valid correspondence region. In Fig. 1)(a), traditional registration resulted in large errors, especially near the resection. While RTR in Fig. 1(b) showed great improvement, the RIME difference image in Fig. 1(c) appeared even flatter.

Table 1 lists the minimum, maximum, mean, and standard deviation of displacement errors for voxels in $R$ which had a valid correspondence in $P$, averaged over the image pairs. We performed one-tailed paired t-tests to evaluate the results and considered $p<0.05$ significant. Only the mean error for RTR significantly decreased compared to SNRR. On the other hand, all RIME error statistics were significantly reduced compared to SNRR. Furthermore, the minimum, mean, and standard deviation of errors for RIME were significantly smaller than those for RTR. The quantitative analysis confirmed the qualitative observations that RIME produced more accurate results than SNRR and RTR.

The estimated indicator function for valid correspondence pixels is shown in Fig. 2, with the true map outlined in green. Typically, errors occurred in the CSF or near the boundary of the brain and background or resection. The dice coefficient was computed for each pair to measure the overlap between the true and estimated maps for valid correspondences, giving an average of 0.98 .

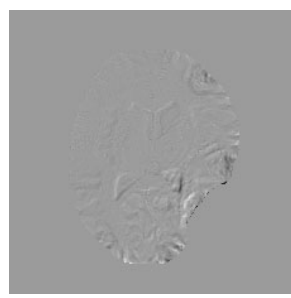

(a)

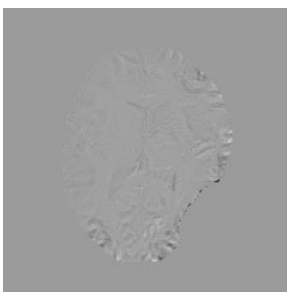

(b)

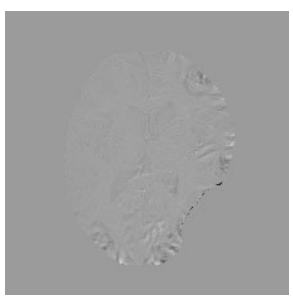

(c)

Fig. 1. Sample difference images between a simulated postresection image and the corresponding warped preoperative image using (a) SNRR, (b) RTR, and (c) RIME

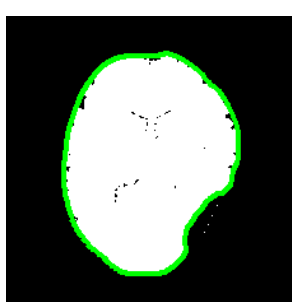

Fig. 2. Outline of true indicator map (green) overlaid on estimated map 
Table 1. Average displacement field errors (in voxels) after synthetic data registration

\begin{tabular}{|c|c|c|c|c|}
\hline Registration Method & Min Error & Max Error & Mean Error & Std Dev Error \\
\hline \hline SNRR & 0.0028 & 3.1952 & 0.3479 & 0.3709 \\
\hline RTR & 0.0017 & 3.1808 & 0.2757 & 0.3083 \\
\hline RIME & 0.0009 & 2.9013 & 0.2272 & 0.2723 \\
\hline
\end{tabular}

\subsection{D Clinical Data}

We applied the registration methods to 6 pairs of preoperative and postresection T1-weighted MR images. First, images were skull stripped using BIS, resampled to $128 \times 128 \times 60$, and affinely aligned. Intensities were renormalized so they could be directly compared. Leave-one-out validation was used, resulting in a training set of about 12500 voxels from 5 images for the resection intensity prior.

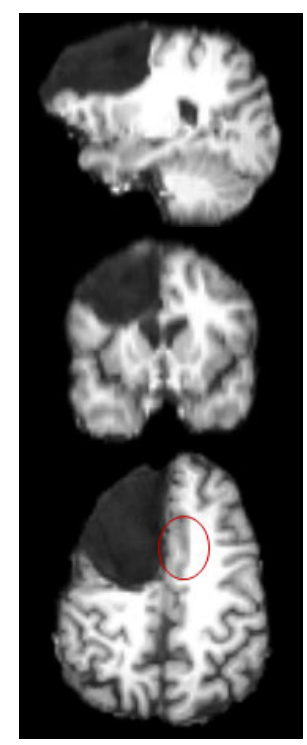

(a)

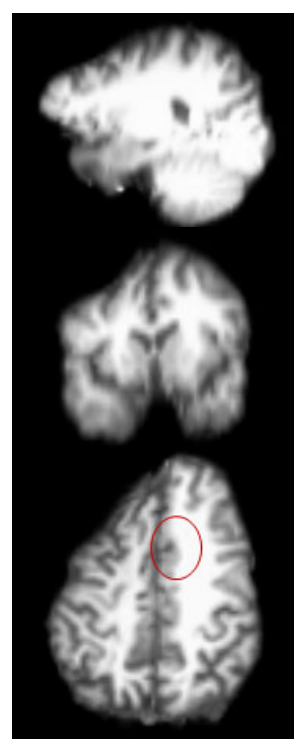

(b)

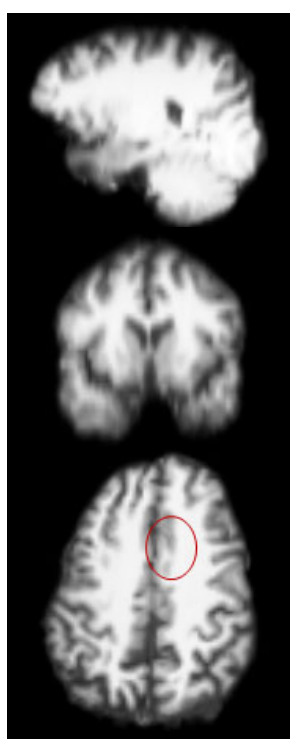

(c)

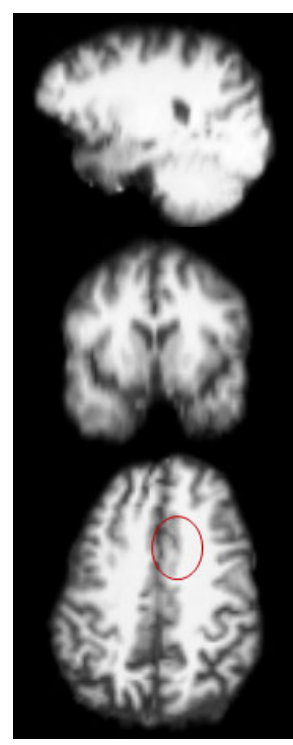

(d)

Fig. 3. Registration of real data. Red ring highlights different results. (a) Postresection image. (b) Warped preoperative image using SNRR. (c) RTR result. (d) RIME result.

Figure 3 displays slices from the registration of real 3D images. The patient had a right frontal lobe resection as seen in Fig. 3(a). Warped preoperative images after SNRR, RTR, and RIME are shown in Figs. 3(b), (c), and (d) respectively. SNRR erroneously deformed the right frontal lobe to match the background to the large resection. This caused misalignment of other features, like the right lateral ventricle and sulci left of the longitudinal fissure. RTR resulted 
in much better alignment of corresponding structures. RIME results showed further improved alignment, such as in the gray matter left of the longitudinal fissure in the axial slice (red ring) and the lateral ventricle in the sagittal image.

We evaluated the registration error by computing the distance between 8 corresponding landmarks spread throughout the brain in each pair of postresection and registered preoperative images. The average errors were $3.99 \mathrm{~mm}$ for affine registration, $2.69 \mathrm{~mm}$ for SNRR, $2.16 \mathrm{~mm}$ for RTR, and $1.27 \mathrm{~mm}$ for RIME. All non-rigid registration methods significantly decreased the error compared to affine (again $p<0.05$ ). The reduced error for RTR compared to SNRR missed significance, while the improvement in RIME error over SNRR was significant.

A sample estimated indicator map is overlaid on a brain with a left temporal lobe resection in Fig. 4. The average dice coefficient measuring the overlap between true and estimated indicator maps for valid correspondences was 0.92 .

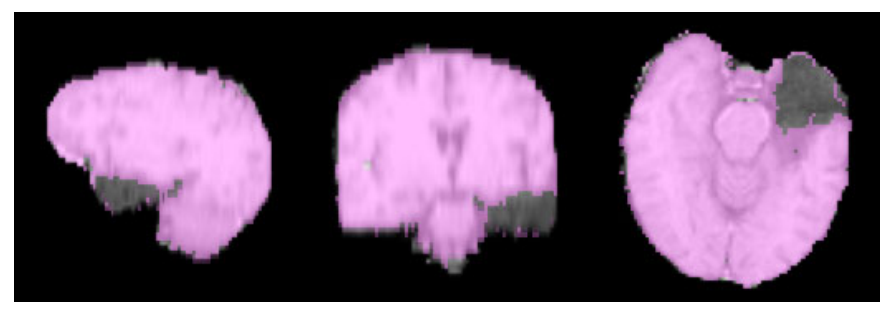

Fig. 4. Estimated indicator map segmenting tissue with correspondences (pink), resection (no color mask), and background (black), overlaid on postresection image

\section{Discussion}

Our proposed RIME method follows a marginalized MAP framework solved using the EM algorithm to estimate both the registration parameters and a "hidden" map indicating the different correspondence regions. The formulation of the problem allows the inclusion of a prior based on voxel intensities in the postresection images given the correspondence label. Our method resulted in significantly improved accuracy compared to a standard non-rigid registration technique (SNRR) and a method using a robust similarity metric (RTR).

Future work will incorporate probability models based on image histograms for the similarity measure to extend the method to intermodality registration. A greater number of labels in the indicator map, for example to represent gray and white matter, may further increase registration accuracy. We will also work to improve the final estimate of the indicator map, which sometimes mislabeled voxels with valid correspondences as resection. This may be due to dark intensities matching the intensity prior better than the similarity term (such as in the CSF) or due to high dissimilarity in intensities near the brain and background border. To simplify the calculations in this paper, we had assumed no prior spatial information for the indicator map. We plan to reincorporate this 
spatial prior to improve the indicator map estimate, which should in turn result in more accurate registration.

\section{References}

1. Kwan, P., Sperling, M.R.: Refractory seizures: Try additional antiepileptic drugs (after two have failed) or go directly to early surgery evaluation? Epilepsia 50, 57-62 (2009)

2. Periaswamy, S., Farid, H.: Medical image registration with partial data. Med. Image Anal. 10, 452-464 (2006)

3. Winterstein, M., Münter, M.W., Burkholder, I., Essig, M., Kauczor, H.U., Weber, M.A.: Partially resected gliomas: Diagnostic performance of fluid-attenuated inversion recovery $\mathrm{mr}$ imaging for detection of progression. Radiology 254(3), 907-916 (2010)

4. Zheng, Y., Yu, J., Kambhamettu, C., Englander, S., Schnall, M.D., Shen, D.: Deenhancing the dynamic contrast-enhanced breast mri for robust registration. In: Metaxas, D., Axel, L., Fichtinger, G., Székely, G. (eds.) MICCAI 2008, Part I. LNCS, vol. 5241, pp. 933-941. Springer, Heidelberg (2008)

5. Zacharaki, E.I., Hogea, C.S., Shen, D., Biros, G., Davatzikos, C.: Non-diffeomorphic registration of brain tumor images by simulating tissue loss and tumor growth. Neuroimage 46(3), 762-774 (2009)

6. Risholm, P., Samset, E., Talos, I.F., Wells, W.: A non-rigid registration framework that accommodates resection and retraction. In: Prince, J.L., Pham, D.L., Myers, K.J. (eds.) IPMI 2009. LNCS, vol. 5636, pp. 447-458. Springer, Heidelberg (2009)

7. Pohl, K.M., Fisher, J., Grimson, W.E.L., Kikinis, R., Wells, W.M.: A bayesian model for joint segmentation and registration. NeuroImage 31(1), 228-239 (2006)

8. Chitphakdithai, N., Duncan, J.S.: Pairwise registration of images with missing correspondences due to resection. In: 2010 7th IEEE International Symposium on Biomedical Imaging: From Nano to Macro, pp. 1025-1028 (2010)

9. Rueckert, D., Sonoda, L.I., Hayes, C., Hill, D.L., Leach, M.O., Hawkes, D.J.: Nonrigid registration using free-form deformations: application to breast $\mathrm{mr}$ images. IEEE Trans Med. Imaging 18(8), 712-721 (1999)

10. Greene, W.H., Chelikani, S., Purushothaman, K., Knisely, J., Chen, Z., Papademetris, X., Staib, L.H., Duncan, J.S.: Constrained non-rigid registration for use in image-guided adaptive radiotherapy. Med. Image Anal. 13(5), 809-817 (2009)

11. Papademetris, X., Jackowski, M., Rajeevan, N., Okuda, H., Constable, R., Staib, L.: BioImage Suite: An integrated medical image analysis suite. Section of Bioimaging Sciences, Dept. of Diagnostic Radiology, Yale School of Medicine, http://www.bioimagesuite.org

12. Arya, K.V., Gupta, P., Kalra, P.K., Mitra, P.: Image registration using robust m-estimators. Pattern Recognition Letters 28, 1957-1968 (2007) 